\title{
Echocardiographic Assessment of Cardiac Resynchronization Therapy
}

\author{
Carlos Eduardo Suaide Silva, Antonio Carlos Pereira Barretto \\ OMNI-CCNI Medicina Diagnóstica/Diagnósticos da América and Instituto do Coração do Hospital das \\ Clínicas - FMUSP - São Paulo, SP - Brazil
}

Heart failure is one of the major causes of mortality, morbidity, and hospitalization in patients older than 60 years, accounting for 1 to $2 \%$ of the total expenditure in the health sector in the United States (approximately 20 billion dollars per year!) ${ }^{1,2}$. Despite the great advances in therapy, the morbidity and mortality rates still remain high ${ }^{3}$. Cardiac resynchronization therapy (CRT) was introduced in the beginning of the 1990's and rapidly developed until its approval in 2001 by the FDA (Food and Drugs Administration) 4 .

In the American Heart Association guidelines, CRT has been considered to be IIA evidence level ${ }^{5}$. Those guidelines were based on 2 large trials: the MUSTIC ${ }^{6}$ and the MIRACLE ${ }^{7}$. In both, the inclusion criteria were similar: a) significant heart failure despite appropriate therapy; b) low ejection fraction; and c) broad QRS with left bundle-branch block pattern (duration>120 ms). Both studies have confirmed that CRT significantly improves symptoms, tolerance to exercise, and quality of life. Nevertheless, 20\% to $30 \%$ of the patients do not improve with $\mathrm{CRT}^{8}$, emphasizing the need for new criteria of patients' selection.

Recent studies have reported that mechanical dyssynchrony is not always related to electrical dyssynchrony ${ }^{9,10}$, and that the presence of ventricular dyssynchrony is the best predictor of a good response to resynchronization therapy. In reality, some patients with broad QRS may not have mechanical dyssynchrony while others with narrow QRS may ${ }^{11-13}$.

Although QRS duration is a good prognostic marker for mortality in patients with heart failure and is present in more than $80 \%$ of the individuals in the 2 months preceding death ${ }^{14,15}$, studies comparing QRS alterations with the clinical outcome of patients seem to show little or no relation to prognosis ${ }^{16}$.

These data suggest that electrocardiography may not be the best complementary diagnostic method for selecting candidates to CRT. Other imaging techniques, particularly the new methods for assessing ventricular function on echocardiography, seem to be better for selecting patients who will best respond to resynchronization therapy.

Both the presence of broad QRS and the signs of interventricular

Mailing address: Carlos Eduardo Suaide Silva - OMNI-CCNI - Rua Cubatão, 726 - 04013-002 - São Paulo, SP - Brazil

E-mail: csuaide@ cardiol.br

Received for publication: 10/20/04

Accepted for publication: 3/4/05

English version by Stela Maris Costalonga dyssynchrony are predictors of hospitalization and severe cardiac events in patients with heart failure $\mathrm{e}^{17,18}$.

\section{Ventricular dyssynchrony}

The mechanism of dyssynchrony includes regional delays of both ventricular contraction and relaxation. The right ventricle contracts during left ventricular telediastole leading to a septal bulging towards the left ventricle. In addition, the delay in the activation of the papillary muscles causes, or worsens, mitral incompetence ${ }^{19}$. All such factors contribute to a reduction in the ejection fraction and a worsening of the clinical symptoms.

Dyssynchrony may be inter- or intraventricular, and echocardiography may evaluate both types through several techniques.

One way of evaluating interventricular dyssynchrony is by measuring the time between ventricular ejections. Conventional Doppler measures the time interval between the $\mathrm{R}$ wave in the electrocardiogram and the beginning of the systolic waves of pulmonary and aortic ejection (fig. 1). A delay greater than 60 ms between those measurements indicates interventricular dyssynchrony. The limitation of such method is that those measurements are not taken simultaneously ${ }^{20-22}$.

Another way to assess delay is through the $M$ mode, in which the time between septal contraction and posterior wall contraction is measured (fig. 2). This time is considered normal when under $130 \mathrm{~ms}^{23}$. In this case, the limitation is the fact that only the middle regions of the septal and posterior walls are assessed.

Intraventricular dyssynchrony is considered one of the most important aspects of electromechanical delay and may be assessed through several echocardiographic techniques. The electromechanical delay has been defined as the time between the beginning of the QRS complex and the peak systolic wave of tissue Doppler (echocardiographic technique that measures the velocity of myocardial motion) in the corresponding myocardial segment (fig. 3).

Guidelines about this subject still lack, and several authors have published studies proposing several indices for diagnosing interand intraventricular dyssynchrony based on tissue Doppler techniques. Yu et al ${ }^{11}$ studied 88 healthy individuals, 67 patients with heart failure (CHF) and narrow QRS ( $<120 \mathrm{~ms})$, and 45 patients with CHF and broad QRS (>120 ms). The authors measured the time between the beginning of QRS and the systolic peak on tissue Doppler (electromechanical delay) in 12 myocardial segments. The authors concluded that the following 2 parameters were indicators 

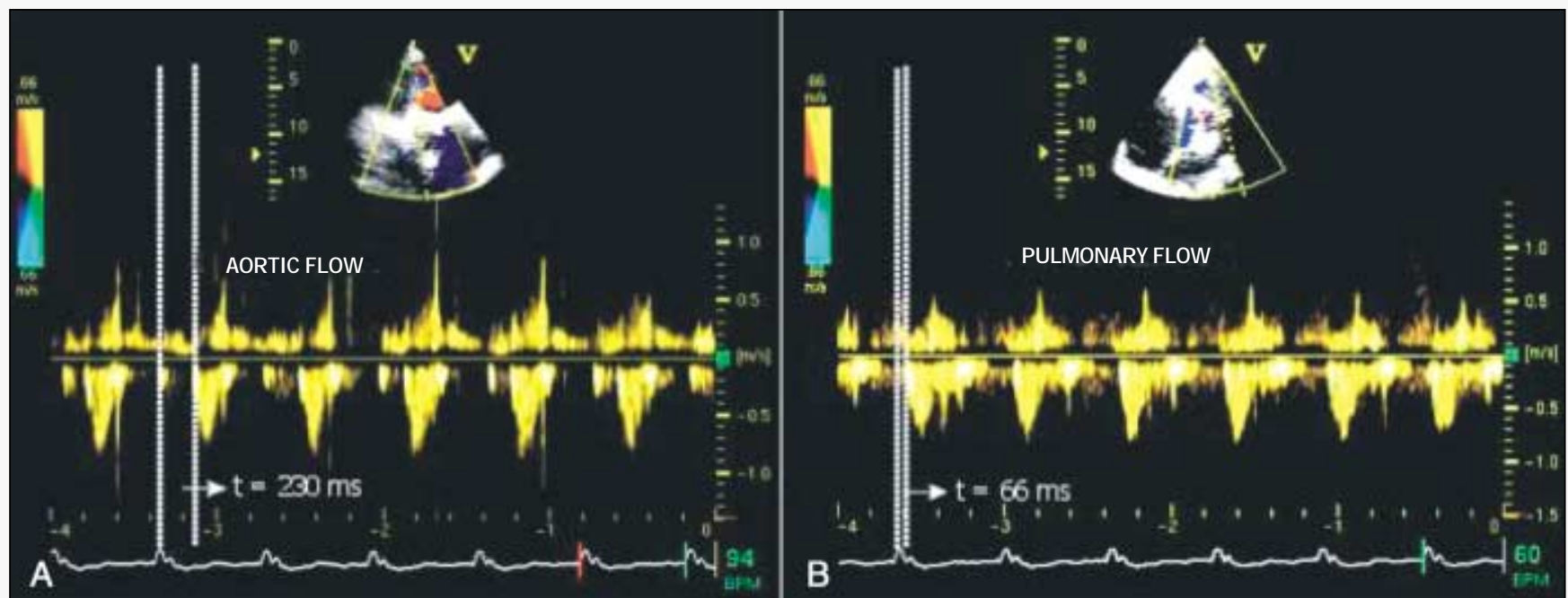

Fig. 1 - Mechanical interventricular delay measured in a patient with heart failure and left bundle-branch block. A) Time between QRS and aortic ejection of $230 \mathrm{~ms}$. B) time between QRS and pulmonary ejection of $66 \mathrm{~ms}$. The difference between those 2 measurements is the mechanical delay between the ventricles (164 ms, in this case).

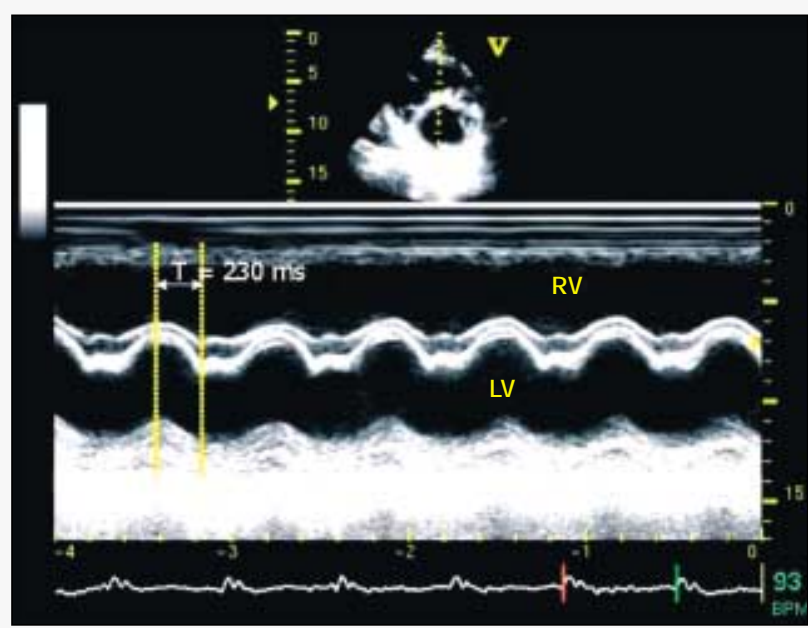

Fig. 2 - Measurement of the interventricular delay in the M mode (RV - right ventricle; LV - left ventricle).

of intraventricular dyssynchrony: the maximum time difference between 2 distinct segments greater than 100 ms; and a 33 standard deviation in the measurement of the 12 segments (named dyssynchrony index). The authors showed lack of dyssynchrony in the healthy group, $73 \%$ of dyssynchrony in patients with $\mathrm{CHF}$ and broad QRS, and, the most interesting, $51 \%$ of dyssynchrony in those with CHF and narrow QRS.

In other studies, dyssynchrony was assessed by using the measure of electromechanical delay in the basal regions of the septal and lateral walls. A difference greater than $60 \mathrm{~ms}$ (septolateral delay) was used as a substantial indicator of dyssynchrony ${ }^{24-25}$ (fig. 4).

The intraventricular electromechanical delay may also be demonstrated by using the Tissue Tracking echocardiographic technique, which represents the integral of the velocity acquired by the tissue Doppler. This technique depicts in color the myocardial motion, from the basis to the ventricular apex. When there is no motion, there is no color. If the systolic and diastolic phases of the cardiac cycle are separately selected, the regions that are contracting may be identified in the respective phases. The regions that are colored in the diastolic phase represent a late myocardial contraction (or postsystolic contraction) and may be easily evi-
The postsystolic motion may be passive or active, in which case it should be called postsystolic contraction or shortening. However, care should be taken with patients with ischemic cardiomyopathy, because that phenomenon is not only a signal of dyssynchrony, but also a marker of ischemia or viability, or both, in akinetic or severely hypokinetic segments. In such cases, it should not be used as a useful criterion to assess a positive response to $\mathrm{CRT}^{26}$.

\section{Automatic detection of dyssynchrony}

Tissue synchronization imaging (TSI) is a new echocardiographic technique that encodes with colors the time intervals between the beginning of the QRS complex and the peak-systolic of tissue Doppler in each myocardial point. It allows the real time visualization of dyssynchronous segments by superimposing the images of such time data over those of 2-dimensional echocardiography. This analysis may be performed in all myocardial segments, but it should be carefully used in the apical segments. The principle is very simple: when the interval up to the peak-systolic (time to peak) is normal, the myocardium is represented in green; when the interval is between 150 and $300 \mathrm{~ms}$, it is represented in yellow; and when it is greater than $300 \mathrm{~ms}$, in red ${ }^{27}$. The result is very interesting and is shown in figure 6 .

\section{Selection of patients for implantation of biventricular pacemaker}

Few published studies have used echocardiography as a tool for choosing patients for biventricular pacemaker implantation. One of them ${ }^{28}$ has assessed 42 individuals with a pacemaker in the RV, 26 of whom had normal ejection fraction, and 16 had impairment of the systolic function and clinical findings of CHF. An electromechanical delay greater than 50 ms identified patients with significant dyssynchrony. No correlation was observed between dyssynchrony and broad QRS in patients with CHF. If only the electrocardiographic criterion had been used for biventricular pacemaker implantation, $44 \%$ of the patients with dyssynchrony would have been excluded, showing the importance of performing echocardiography.

More studies in this area are required to definitively validate that 


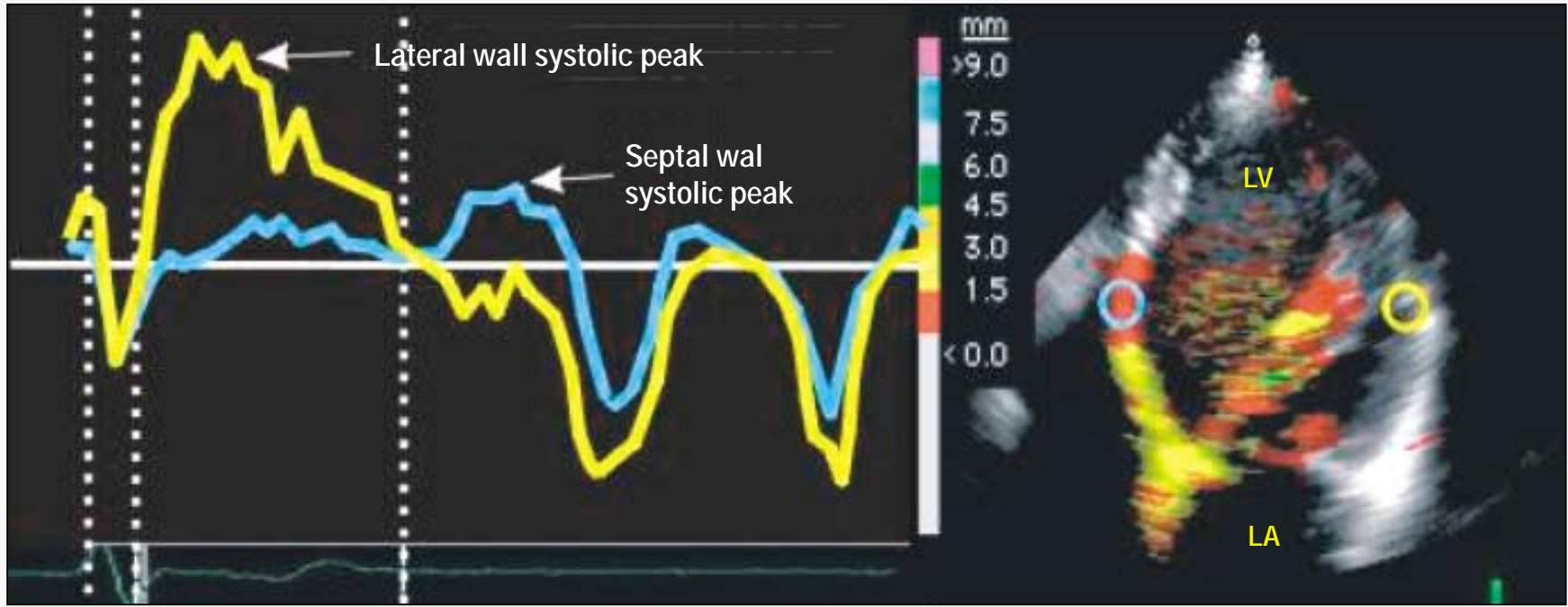

Fig. 3 - Quantification of the intraventricular electromechanical delay on tissue Doppler. In this example, the blue and yellow curves represent the velocities of myocardial motion in the middle regions of the septal and lateral walls, respectively (LA - left atrium; LV - left ventricle).

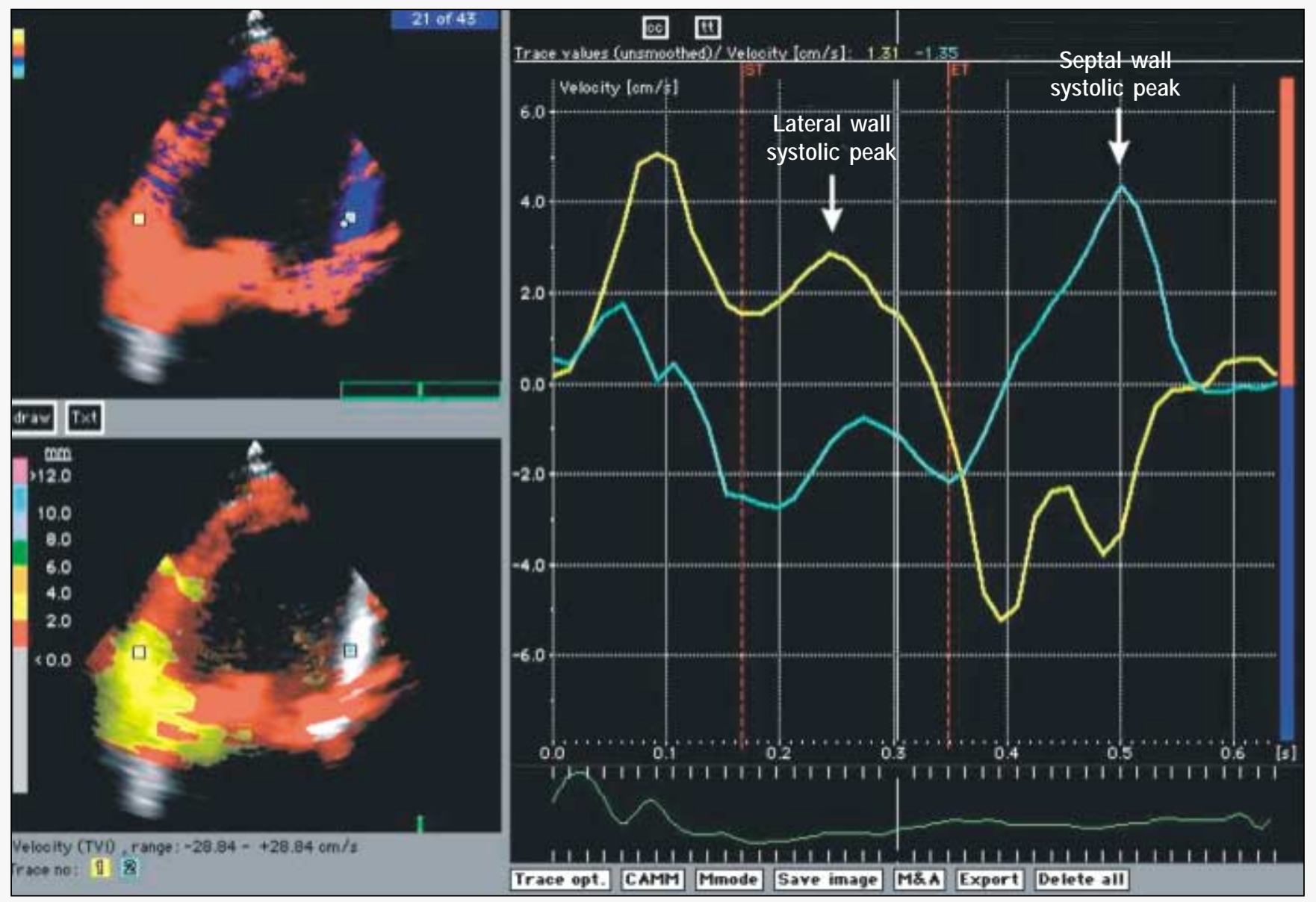

Fig. 4 - Measure of the electromechanical delay in the basal regions of the septal (green curve - $500 \mathrm{~ms}$ ) and lateral (yellow curve - $235 \mathrm{~ms}$ ) walls. A difference greater than $60 \mathrm{~ms}$ is highly indicative of intraventricular dyssynchrony. The red dotted lines delimitate the ventricular ejection period.

methodology. This review, however, suggests that the criteria for biventricular pacemaker implantation should be revised and tissue Doppler echocardiography should be used for selecting such patients.

\section{Where should the electrode be implanted?}

Tissue Doppler may help in determining the ideal site for implanting the electrode within the coronary sinus.
The best site for implantation, ie, the site where the best ventricular response is obtained (gain in ejection fraction), has already been documented as that with the greatest electromechanical delay. This site is in the lateral wall in 35\% of the cases, in the anterior and posterior walls in $26 \%$ and $23 \%$, respectively, and rarely in the inferior and septal walls $(16 \%)^{29}$.

The objective of the resynchronization therapy is to activate the site with the greatest electromechanical delay; therefore, the 


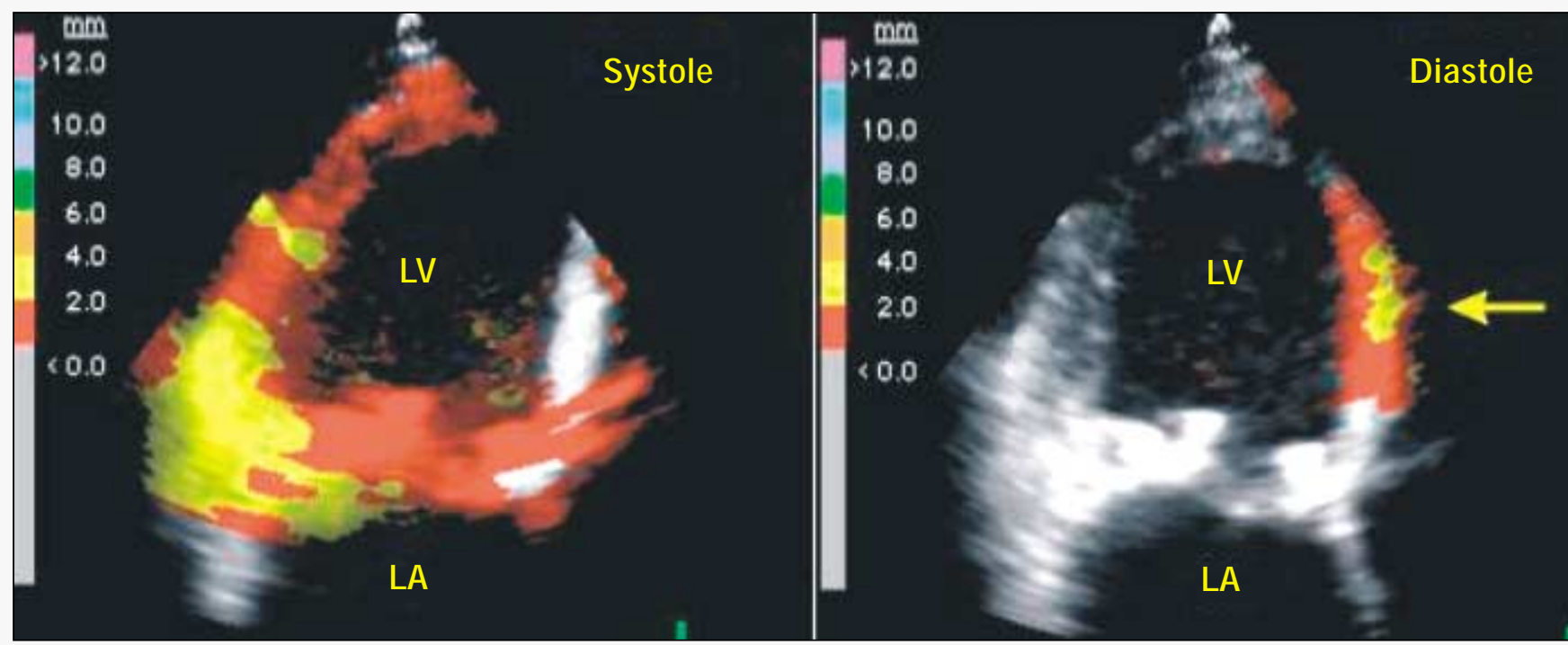

Fig. 5 - Tissue Tracking technique showing the area of late contraction (arrow) of the interventricular septum.

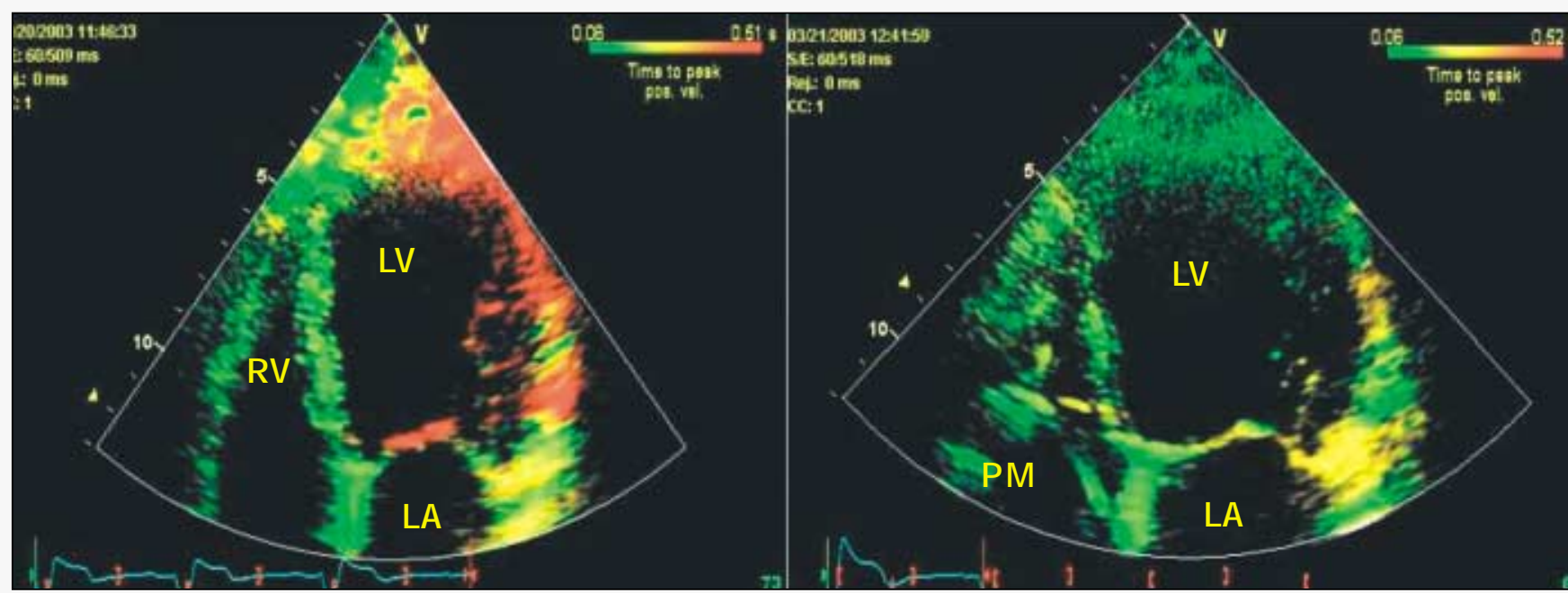

Fig. 6 - Left: Apical 4 -chamber view showing electromechanical delay in the left ventricular lateral wall (red). Right: The same patient after resynchronization showing an improvement in the color pattern (green), representing the reestablishment of contractile synchrony. LA - left atrium; PM - pacemaker electrode; RV - right ventricle; LV - left ventricle.

determination of such site is directly linked to a successful procedure. And, in fact, Ansalone et $\mathrm{al}^{29}$ have demonstrated that the best result was obtained in patients whose electrodes were implanted according to the site with the greatest electromechanical delay, determined on echocardiography.

\section{Echocardiographic markers indicating improvement with CRT}

The most evident signs of improvement after biventricular pacemaker implantation are the increase in ejection fraction, the decrease in the degree of mitral incompetence, and the regression in ventricular remodeling. However, echocardiography may provide the following less evident markers: a) an improvement in the atrioventricular activation assessed through the increase in the time velocity integral of the aortic flow and extension of the time of diastolic filling (assessed through mitral flow) in 10 to $20 \%$; b) reversion of the interventricular electromechanical delay assessed on tissue Doppler. The MIRACLE study reported a $19 \%$ reduction in that marker ${ }^{30}$. Yu et al ${ }^{31}$ reported a complete regression in the great interventricular 4 delay between the free wall of the RV and the lateral wall of the LV after CRT; and c) intraventricular resynchronization. Several studies have confirmed the normalization of the intraventricular delay by using the different techniques above cited ( $M$ mode, pulsed Doppler of the outflow tracts, and tissue Doppler) ${ }^{32-35}$.

\section{Conclusion}

Cardiac resynchronization therapy has been defined by studies involving a reduced number of patients as an excellent therapeutic option for patients with heart failure. However, approximately $30 \%$ of the cases have not responded adequately when the current electrocardiographic criteria of indication were used. The evidence above cited confirms that echocardiography seems to be the ideal complementary method to identify those patients who will be effectively beneficiated by CRT. However, great studies definitively validating the method are yet to be carried out.

In addition to the quantitative diagnosis of resynchronization, the new echocardiographic techniques based on tissue Doppler (Tissue Tracking, Strain Rate, and TSI) may aid in choosing the best site for pacemaker electrode implantation with important benefits for the procedure and in following up the patients in a noninvasive form. 


\section{References}

1. American HeartAssociation. New Medicine Reports 1997; 1999 Heart and Stroke Statistical U pdate. Dallas, TX: American Heart Association.

2. Cleland J GF. The heart failure epidemic: exactly how big is it? Eur HeartJ 2001; 22:623-6.

3. Zannad F, Briancon $S$, J uillière $Y$ et al. Incidence, clinical and etiologic features, and outcomes of advanced chronic heart failure: the EPICAL study. J Am Coll Cardiol 1999; 33: 734-42.

4. Abraham WT, Hayes DL. Cardiac resynchronization therapy for heart failure. Circulation 2003; 108: 2596-603.

5. Gregoratos G, Abrams J , Epstein AE et al. ACC/AHA/NASPE 2002 guideline update for implantation of cardiac pacemakers and antiarrhythmia devices. J Am Coll Cardiol 2002; 40: 1703-19.

6. Cazeau S, Leclercq C, Lavergne T et al. Effects of multisite biventricular pacing in patients with heart failure and intraventricular conduction delay. N Engl J Med 2001; 344:873-80.

7. Abraham WT, Fisher WG, Smith $\mathrm{AL}$ et al. Cardiac resynchronization in chronic heart failure. N EnglJ Med 2002; 346: 1845-53.

8. Leclercq $C$, Kass DA. Retiming the failing heart: principles and current clinical status of cardiac resynchronization. J Am Coll Cardiol 2002; 39: 194-201.

9. Leclercq C, Faris O, Tunin $\mathrm{R}$ et al. Systolic improvement and mechanical resynchronization does not require electrical synchrony in the dilated failing heart with left bundle-branch block. Circulation 2002; 106: 1760-3.

10. Kass DA. Predicting cardiac resynchronization response by QRS duration: the long and short of it. J Am Coll Cardiol 2003; 42: 2125-7.

11. Yu CM, Lin H, Zhang Q. High prevalence of left ventricular systolic and diastolic asynchrony in patients with congestive heart failure and normal QRS duration. Heart 2003; 89: 54-60.

12. Bleeker GB, Schalij MI, Molhoek SG et al. Relationship between QRS duration and left ventricular dyssynchrony in patients with end-stage heart failure. J Cardiovasc Electrophysiol 2004; 15: 544-9.

13. Achilli A, Sassara M, Ficili S et al. Long-term effectiveness of cardiac resynchronization therapy in patients with refractory heart failure and "narrow" QRS. J Am Coll Cardiol 2003; 42: 2117-24.

14. Iuliano S, Fisher SG, Karasik PE, Fletcher RD, Singh SN. QRS duration and mortality in patients with congestive heart failure. Am HeartJ 2002; 143: 1085-91.

15. Wilensky RL, Yudelman P, Cohen Al et al. Serial electrocardiographic changes in idiopathic dilated cardiomyopathy confirmed at necropsy. AmJ Cardiol 1988; 62: 276-83.

16. Sun JP, Chinchoy $E$, Donal $E$ et al. Evaluation of ventricular synchrony using novel Doppler echocardiographic indices in patients with heart failure receiving cardiac resynchronization therapy. J Am Soc Echocardiogr 2004;17: 845-50.

17. Venkateshwar K, Gottipaty VK, Krelis SP, Lu F, Spencer EP, Shusterman V, Weiss $\mathrm{R}$, Brode $S$, White A, Anderson KP, White BG, Feldman AM, For the VEST investigators: The resting electrocardiogram provides a sensitive and inexpensive marker of prognosis in patients with chronic congestive heart failure. J Am Coll Cardiol 1999, 33: 145 (Abstract).

18. Bode-Schnurbus $L$, Bocker D, Block M et al. QRS duration: a simple marker for predicting cardiac mortality in ICD patients with heart failure. Heart 2003; 89: 1157-62.

19. BaxJJ, Ansalone G, Breithardt OA et al. Echocardiographic evaluation of cardiac resynchronization therapy: ready for routine clinical use? A critical appraisal. J Am Coll Cardiol 2004; 44: 1-9.
20. Kanzaki H, J acques D, Sade LE, Severyn DA, Schwartzman D, Gorcsan 」 3rd. Regional correlation by color-coded tissue Doppler to quantify improvements in mechanical left ventricular synchrony after biventricular pacing therapy. Am J Cardiol 2003; 92: 752-75.

21. Porciani MC, Puglisi A, Colella A et al., on behalf of the InSync Italian Registry Investigators. Echocardiographic evaluation of the effect of biventricular pacing: the InSync Italian Registry. Eur Heart) Suppl 2000; 2(Suppl J): J 23-30.

22. Rouleau F, Merheb M, Geffroy S et al. Echocardiographic assessment of the interventricular delay of activation and correlation to the QRS width in dilated cardiomyopathy. Pacing Clin Electrophysiol 2001; 24: 1500-6.

23. Pitzalis MV, Iacoviello M, Romito $R$ et al. Cardiac resynchronization therapy tailored by echocardiographic evaluation of ventricular asynchrony. J Am Coll Cardiol 2002; 40: 1615-22

24. Bax J J, Molhoek SG, van Erven L et al. Usefulness of myocardial tissue Doppler echocardiography to evaluate left ventricular dyssynchrony before and after biventricular pacing in patients with idiopathic dilated cardiomyopathy. Am J Cardiol 2003; 91: 94-7.

25. BaxJJ, Marwick TH, Molhoek SG et al. Left ventricular dyssynchrony predicts benefit of cardiac resynchronization therapy in patients with end-stage heart failure before pacemaker implantation. Am J Cardiol 2003; 92: 1238-40.

26. Mele D, Pasanisi G, Heimdal A et al. Improved recognition of dysfunctioning myocardial segments by longitudinal strain rate versus velocity in patients with myocardial infarction. J Am Soc Echocardiogr 2004: 313-21.

27. Penicka M, Bartunek J, De Bruyne B et al. Improvement of left ventricular function after cardiac resynchronization therapy is predicted by tissue Doppler imaging echocardiography. Circulation 2004; 109: 978-83.

28. Bordachar P, Garrigue S, Lafitte $S$ et al. Interventricular and intra-left ventricular electromechanical delays in right ventricular paced patients with heart failure: implications for upgrading to biventricular stimulation. Heart 2003; 89: 1401-05.

29. Ansalone G, Giannantoni P, Ricci R, Trambaiolo P, Fedele F, Santini M. Doppler myocardial imaging to evaluate the effectiveness of pacing sites in patients receiving biventricular pacing. J Am Coll Cardiol 2002; 39: 489-499.

30. Kindermann M, Frolhig G, Doerr T, Schieffer H. Optimizing the AV delay in DDD pacemaker patients with high degree AV block: mitral valve Doppler versus impedance cardiography. Pacing Clin Electrophysiol 1997; 20: 2453-62.

31. Yu CM, Chau E, Sanderson JE et al. Tissue Doppler echocardiographic evidence of reverse remodeling and improved synchronicity by simultaneously delaying regional contraction after biventricular pacing therapy in heart failure. Circulation 2002; 105: 438-45

32. Breithardt OA, Stellbrink C, Kramer AP et al. Echocardiographic quantification of left ventricular asynchrony predicts an acute hemodynamic benefit of cardiac resynchronization therapy. J Am Coll Cardiol 2002; 40: 536-45.

33. Kawaguchi M, Murabayashi T, Fetics BJ et al. Quantitation of basal dyssynchrony and acute resynchronization from left or biventricular pacing by novel echo-contrast variability imaging. J Am Coll Cardiol 2002; 39: 2052-8.

34. Yu CM, Fung WH, Lin $\mathrm{H}$ et al. Predictors of left ventricular reverse remodeling after cardiac resynchronization therapy for heart failure secondary to idiopathic dilated or ischemic cardiomyopathy. Am J Cardiol 2003; 91: 684-8.

35. Breithardt OA, Stellbrink $C$, Herbots $L$ et al. Cardiac resynchronization therapy can reverse abnormal myocardial strain distribution in patients with heart failure and left bundle branch block. J Am Coll Cardiol 2003; 42: 486-94. 